\section{Determinación de la profundidad del paladar en niños con respiración bucal de 6-8 años de edad}

Nieto P, Acosta J, Meneses A. Determinación de la profundidad del paladar en niños con respiración bucal de 6-8 años de edad. Rev Estomatol Herediana 2005;15(1): 50 - 53

\section{RESUMEN}

El propósito de este trabajo fue evaluar la influencia de la respiración bucal con respecto a la profundidad del paladar. La población estuvo conformada por niños de 6-8 años de edad pertenecientes al CE Fe y Alegría $\mathrm{N}^{\circ} 2$ del distrito de San Martín de Porres, a quines se les realizó exámenes clínicos donde se midió la profundidad del paladar utilizando como instrumento una plantilla milimetrada, previamente calibrada. Se utilizaron pruebas estadísticas de distribución de frecuencias, chi-cuadrado, t de Student, y U de Mann-Whitney. El 29.3\% de la población presentó respiración bucal, siendo la profundidad palatina promedio hallada a nivel de molares y caninos de 17,38 mm y 6,72 mm, respectivamente. Y en grupo control fue de 7,83 $\mathrm{mm}$ y $16,81 \mathrm{~mm}$ respectivamente. La profundidad palatina a nivel de molares fue mayor y a nivel de caninos menor en los respiradores bucales. Existiendo una una asociación entre la respiración bucal y la variación de la profundidad palatina a nivel de molares.

Palabras clave: RESPIRACIÓN POR LA BOCA / PALADAR DURO. anatomía \& histología.

Determination of the palatal depth in mouth breather children of 6-8 years of age in comparison with a control group

ABSTRACT

The purpose of this study was to evaluate the influence of mouth breathing in palatal depth. The palatal depth was evaluated in two levels: canines and second primary molars. The values were compared between the mouth breather group and the control group. The sample consisted of 99 children of 6-8 years old who studied at the school "Fe y Alegria $N^{\circ} 2$ ". Dental exams were performed and the palatal depth was measured with a millimetre ruler(specially designed by the authors of the study). Chi-square test ,t-Student and U- Mann Whitney were used as statistical tests. $29.3 \%$ of the sampled population were mouth breathers. The mean of the palatal depth in the mouth breather group at the level of canines and molars was $17.38 \mathrm{~mm}$ and 6.72 , respectively. The mouth breathers showed a higher palatal depth at the level of molars than the control group. The control group showed a higher palatal depth at the level of canines than the mouth breather group. Results show that there exists an association between the oral breathing and the variations in the palate depth at molars level.

Keywords: MOUTH BREATHING / HARD PALATE. anatomy \& histology.

\section{Patricia Nieto Perea ${ }^{1}$ Jorge Acosta Quiñones ${ }^{2}$ Abraham Meneses López ${ }^{2}$}

${ }^{1}$ Cirujano dentista

${ }^{2}$ Docente del Departamento Académico de Estomatología del Niño y del Adolescente. Facultad de Estomatología. Universidad Peruana Cayetano Heredia

\section{Correspondencia}

Patricia Nieto Perea,

Calle Jose C. Arnao 110-201, Urb. Ingeneria, Lima 31 - Perú.

E-mail: Panp82@yahoo.es

Aceptado para publicación : 25 de junio del 2005

\section{Introducción}

El desarrollo y maduración de la función bucofaríngea son procesos complejos que intervienen de manera importante en el crecimiento craneofacial y la fisiología oclusal. La actividad muscular anormal tiene efectos nocivos en el desarrollo de las estructuras de la boca y en la maduración de la función estomatognática. Dichos efectos pueden incluir malposición dentaria, anormalidad de los patrones de respiración, anomalías del habla, desequilibrios funcionales de músculos de la cara y pro- blemas psicológicos (1).

La respiración, alimentación materna, masticación, deglución y la pronunciación de sonidos forman parte del sistema funcional neuromuscular; siendo el acto respiratorio, de gran importancia para estimular y mantener un balance durante y después del desarrollo craneofacial (2,3). Linder-Aronson (4) encontraron que en la respiración nasal existe un componente de respiración bucal y viceversa. Concluyendo que el respirador bucal es aquel que la mayor parte del tiempo respira por la boca. Las características faciales externas de este tipo de pacientes han adoptado diversos nombres como: fascie adenoidea, fascie microrrinica y síndrome de obstrucción respiratoria (5-11) O’Ryan et al. (12) en 1982 concluyeron que al existir falta de estimulación muscular normal de la lengua y, debido a presiones mayores sobre los arcos de los labios y buccinador; los segmentos bucales del maxilar superior se estrechan dando un maxilar en forma de "V" y una bóveda palatina elevada. Proffit (13) refiere que el conservar las vías aéreas abiertas 
puede afectar las posiciones vertical y horizontal de los dientes y además influir en el crecimiento de los maxilares. Faria (14) refiere que la respiración bucal interfiere en la posición anteroposterior del maxilar debido a una reducción del flujo de aire en la cavidad nasal. Esto produce hipoplasia de los senos nasales y paranasales y a una reducción de la presión ejercida de la lengua contra el paladar originando modificaciones en su anatomía.

Existen dos métodos para medir la profundidad palatina, el directo intraoral y el indirecto a través de modelos de estudio o análisis cefalométrico. Además se pueden tomar como referencia la segunda molar decidua superior o la primara molar permanente superior.

El presente estudio busca establecer si existe una influencia de la respiración bucal con respecto a la profundidad del paladar en dos sectores: caninos y segundas molares deciduas superiores, comparando estos valores con un grupo control.

\section{Materiales y método}

Se seleccionaron 99 niños del Centro Educativo “Fe y Alegría N²” del distrito de San Martín de Porres de un total de 129 niños de 6 a 8 años de edad sin antecedentes de hábitos de succión, alteraciones verticales o transversales evidentes, problemas congénitos o sistémicos, traumas a nivel orofacial y que no tuvieran historia de tratamiento ortodóntico.

El procedimiento para diagnosticar a los respiradores bucales fue el siguiente :

1. Con la asesoría de otorrinolaringólogos del Hospital Nacional

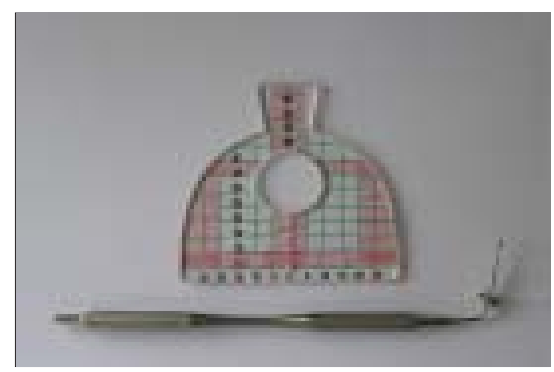

Figura 1. Instrumento diseñado para medir la profundidad palatina.
Cayetano Heredia se confeccionó un cuestionario dirigido a los padres de familia, con preguntas dirigidas a conocer la frecuencia del hábito, su duración y en que momento del día se producía su práctica, las cuales sirvieron como ayuda diagnóstica.

2. Pruebas clínicas :

- Se observó el empañamiento de un espejo bucal que era posicionado a nivel de los orificios nasales.

- Se pidió retener una pequeña cantidad de agua por un periodo de tres minutos (16). Al no poder retener el agua se consideró como un posible respirador bucal.

- Se pidió al niño que cierre los labios y haga una inspiración forzada por la nariz, y se observó la dilatación de las aletas de la nariz indicándonos la salida o no del aire inspirado.

3. Se evaluó la presencia de características faciales típicas de un respirador bucal como: cara estrecha, narinas pequeñas poco desarrolladas.

Una vez diagnosticados se conformaron los grupos de respiradores bucales (RB) y el grupo control (GC).

Se diseñó un instrumento para medir la profundidad palatina, que consistía en una plantilla milimetrada de acrílico con forma de arcada dentaria. En sus superficie presenta líneas guía horizontales de color verde espaciadas a $5 \mathrm{~mm}$ y líneas verticales de color rojo espaciadas a $1 \mathrm{~mm}$. A su vez presenta una línea central con orificios que permiten el ingreso de una sonda periodontal milimetrada que permite medir de la profundidad del paladar (Fig. 1).

La confiabilidad del instrumento se demostró en un estudio piloto donde

Tabla 1. Profundidad del paladar a nivel de caninos en respiradores bucales y el grupo control

\begin{tabular}{lll}
\hline & X & D.E. \\
\hline Respirador bucal & 6,72 & 1,16 \\
Grupo control & 7,83 & 1,01 \\
Total & 7,51 & 1,16 \\
\hline
\end{tabular}

se compararon las medidas intraorales registradas en cinco pacientes con sus respectivos modelos de estudio no hallándose diferencias en las medidas. Se realizó un segundo estudio preliminar para evaluar las medidas tomando como punto de referencia la superficie distal de la segundad molar decidua superior y la superficie mesial de la primera molar permanente superior. Mediante un análisis de varianza, no se encontraron diferencias significativas al registrar las medidas en ambos puntos de referencia $(\mathrm{p}=0,368)$.

Se realizó un análisis multivariado de distribución de frecuencias para evaluar las variables: profundidad del paladar, respiración bucal, y sexo. Se utilizó la prueba t de Student y U de MannWhitney para determinar la variabilidad de los resultados de la profundidad palatina entre los respiradores bucales y el grupo control. Los cálculos se realizaron en el programa SPSS12.0.

\section{Resultados}

De la muestra estudiada, 29 niños presentaron respiración bucal (29,3\%), y 70 niños no presentaron respiración bucal $(70,7 \%)$ conformando el grupo control.

La profundidad palatina promedio a nivel de caninos del total de la muestra fue de 7,51 mm (DE=1,16 mm). En los RB fue de $6,72 \mathrm{~mm}$ ( $\mathrm{DE}=1,16 \mathrm{~mm})$, y en el GC fue de 7,83 mm (DE=1,0 mm) (Tabla 1).

La profundidad palatina promedio a nivel de molares del total de la muestra fue de 16,98 mm (DE=1,13 mm). En los RB fue de 17,38 mm (DE=0,90 mm), y en el GC fue de 16,81 mm (DE=1,18 mm) (Tabla 2).

Tabla 2. Profundidad del paladar a nivel de molares en respiradores bucales y el grupo control

\begin{tabular}{lll}
\hline & $\mathrm{X}$ & $\mathrm{D} . \mathrm{E}$. \\
\hline Respirador bucal & 17,38 & 0,90 \\
Grupo control & 16,81 & 1,18 \\
Total & 16,98 & 1,13 \\
\hline
\end{tabular}


Analizados los resultados según sexo, se encontró: profundidad palatina promedio a nivel de caninos en el grupo de mujeres fue de 7,57 mm (DE=1,12 mm), y en el grupo de hombres fue de 7,43 $\mathrm{mm}(\mathrm{DE}=1,22 \mathrm{~mm})$ (Tabla 3).

La profundidad palatina promedio a nivel de molares en el grupo de mujeres fue de $16,79 \mathrm{~mm}$ ( $\mathrm{DE}=1,10 \mathrm{~mm})$, y en el grupo de hombres fue de 17,20 mm (DE=1,15 mm) (Tabla 4).

Al comparar los valores promedio de la profundidad palatina a nivel de caninos en ambos grupos de estudio utilizando la prueba t de Student y U de Mann-Whitney se halló un $\mathrm{p}=0,00$ estadísticamente significativo con un intervalo de $-1,60$ a -0,61.

Al comparar el análisis de los promedios de la profundidad del paladar a nivel de molares en ambos grupos de estudio utilizando la prueba t de Student y U de Mann- Whitney se halló un $\mathrm{p}=0,012$ y 0,034 altamente significativo respectivamente con un intervalo de 0,13 a 1,00 (Tabla 5).

\section{Discusión}

Se evaluó la profundidad del paladar a nivel de caninos y segundas molares deciduas en un grupo de niños con respiración bucal y un grupo control con la finalidad de determinar si un niño respirador bucal presentaba un paladar mas profundo en comparación con los no respiradores bucales. Diversos autores, como Rogelio (16), Linder

Tabla 3. Profundidad del paladar a nivel de caninos según sexo.

\begin{tabular}{lll}
\hline & $\mathrm{X}$ & $\mathrm{D} . \mathrm{E}$. \\
\hline Femenino & 7,57 & 1,12 \\
Masculino & 7,43 & 1,22 \\
Total & 7,51 & 1,16 \\
\hline
\end{tabular}

Tabla 4. Profundidad del paladar a nivel de molares según sexo.

\begin{tabular}{lll}
\hline & $\mathrm{X}$ & D.E. \\
\hline Femenino & 16,79 & 1,10 \\
Masculino & 17,20 & 1,15 \\
Total & 16,98 & 1,13 \\
\hline
\end{tabular}

Tabla 5. Comparación entre las medias de la profundidad palatina a nivel de caninos y molares en los respiradores bucales y el grupo control.

\begin{tabular}{|c|c|c|c|c|c|c|c|c|}
\hline & \multicolumn{2}{|c|}{$\begin{array}{l}\text { Respiradores } \\
\text { Bucales }\end{array}$} & \multicolumn{2}{|c|}{$\begin{array}{l}\text { Grupo } \\
\text { control }\end{array}$} & \multicolumn{2}{|c|}{ Intervalo } & \multirow[b]{2}{*}{ Min } & \multirow[b]{2}{*}{ Max } \\
\hline & $X$ & D.E. & $X$ & D.E. & $\mathrm{p}(*)$ & $\mathrm{p}(+)$ & & \\
\hline A nivel de caninos & 6,72 & 1,16 & 7,83 & 1,01 & 0,00 & 0,00 & $-1,60$ & $-0,61$ \\
\hline A nivel de molares & 17,38 & 0,90 & 16,81 & 1,18 & 0,012 & 0,034 & 0,13 & 1,00 \\
\hline
\end{tabular}

(*)t de Student • (+)U de Mann-Whitney

Aronson (17), Linder Aronson y Backstrom (18), Hanson y Cohen (19) y Trask et al.(20) concuerdan en que la profundidad palatina es mayor en los sujetos respiradores bucales. El presente estudio halló que los respiradores bucales presentan un paladar más profundo a nivel de molares $(17,38 \mathrm{~mm})$ que en el grupo de no respiradores bucales (16,81 mm).

Linder Aronson (17) en 1963 encontró una profundidad palatina de 14,2 mm en un grupo control y una profundidad palatina de $15,9 \mathrm{~mm}$ en respiradores bucales. Freitas (2) en 2001 mediante el compás tridimensional de Korkhaus y posicionándolo a nivel de la fosa central de las segundas molares deciduas, encontró en los niños respiradores bucales y no reparadores bucales en dentición mixta, una profundidad media palatina de 11,96 y 10,23 mm respectivamente.

Bresolin (15) en 1983 y Trasks y Shapiro (20) en 1987 utilizando radiografías cefalométricas, encontraron una profundidad media palatina de $15,7 \mathrm{~mm}$, 16,72 mm en los respiradores bucales, y 14,5 mm, 15,17 mm en el grupo control.

En este estudio se registraron medidas de la profundidad del paladar a nivel de los caninos y las segundas molares deciduas con la finalidad de determinar en que sector del paladar se presenta la variación. Otros estudios no realizaron medidas a nivel de los caninos. La profundidad media canina encontrada en el grupo respirador bucal y

\section{Referencias bibliografías}

1. Stuart D. Hábitos que afectan el crecimiento y desarrollo dentales y maxilofaciales. Clínicas Odontológicas de Norteamérica. 1995;4:861-9.

2. Freitas F, Bastos E, Primo S. Evaluation of the palate dimensions of patients with perennial allergic rhinitis. Int $\mathrm{J}$ of Paediatric Dentistry.2001;11:365-71.

3. Defabjanis P. Impact of nasal airway obstruction on dentofacial dentofacial development and sleep disturbances in children: preliminary notes. J Clin Pediatr Dent.2003; 27(2):95-100.

4. Linder-Aronson. The mouth breather and the muscular seals of the oral cavity. Proc Roy Soc Med. 1988;51(4):279-82.

5. Tourme L. The long face syndrome and impairment of the nasopharyngeal airway. Angle orthod.1989; 60(3):167-76.

6. Moyers RE. Manual de ortodoncia. Cuarta edición. Editorial Panameri- 


\section{cana.1992}

7. Graber T. Ortopedia dentofacial con aparatos funcionales. 2da edición. Editorial Harcourt Brace. Barcelona. 1998:5-11.

8. Warren D, Hairfield W, et al. Nasal airway impairment: The oral response in cleft palate patients. AM. J. Orthod.1991; 99(4):346-53.

9. Warren D. A quantitative technique for assesing nasal airway impairment. Am. J. Orthod.1984; 86(4): 306-12.

10. Berkinshaw E. The effect of methodology on the determination of nasal ressistance. Am J Orthod Dentofacial Orthop. 1987;92:329-35.

11. Asociación de enfermedades nasorespiratorios, pie plano, escoliosis y problemas psicopedagógicos en niños respiradores bucales con maloclusión. [Editorial].Rev. Cubana Orto.1986;1(2):93-100
12. Bresolin D, Shapiro G.Facial Characteristic of children who breath through the mouth. Pediatrics 1984;73: 622-5.

13. Proffit W.Contemporary Orthodontics, ed 2. St Louis. Mosby-Year Book.1993

14. Faria P, Ruellas A, Matsumoto M, Anselmo-Lima W, et al.Dentofacial Morphology of mouth breathing Children. Braz Dent J. 2002; 13(2):129-32.

15. Bresolin D. Mouth Breathing in allergic children: its relationship to dentofacial development. Am J Orthod and Dentofacial Orthop. 1998; 83:334-40.

16. Rogério A, Bommarito S, Mandetta S, Trevisi A.C. Avaliaòão da profundidade palatina em pacientes com diferentes tipos faciais. Revista Odonto $\mathrm{N}^{0}$ 20, 2002, 1ra edicion;
Editora da Universidade Metodista de São Paulo.

17. Linder-Aronson S. Dimensions of face and palate in nose breathers and habitual mouth breathers. Odont. Revy. 1963 Feb.; 14: 187-200.

18. Linder-Aronson S, Backstrom A. a comparison between mouth and nose breathers with respect to occlusion and facial dimensions. A biometric study. Odont. Revy 11:343-76,1960

19. Hanson M, Cohen M. Effects of form and function on swallowing and the development dentition.Am. J. Orthod.1973;64:63-81.

20. Trask G, Shapiro G, Shapiro P. the effects of perennial allergic rhinitis on dental and skeletal development: A comparison of sibling pairs. Am J of orthodontics and Dentofacial Orthopedicts.1987;92:286-93. 